\title{
Overexpression of the androgen receptor in human hepatoma cells and its effect on fatty acid metabolism
}

\author{
TATSUO KANDA $^{1}$, XIA JIANG ${ }^{1,3}$, MASATO NAKAMURA $^{1}$, YUKI HAGA ${ }^{1}$, REINA SASAKI $^{1}$, \\ SHUANG WU ${ }^{1}$, SHINGO NAKAMOTO ${ }^{1,2}$, FUMIO IMAZEKI ${ }^{1}$ and OSAMU YOKOSUKA ${ }^{1}$
}

\author{
Departments of ${ }^{1}$ Gastroenterology and Nephrology, and ${ }^{2}$ Molecular Virology, Graduate School of Medicine, \\ Chiba University, Chiba 260-8670, Japan; ${ }^{3}$ Department of General Surgery, The First Hospital of Hebei Medical University, \\ Shijiazhuang, Hebei 050031, P.R. China
}

Received February 6, 2016; Accepted February 3, 2017

DOI: $10.3892 / 01.2017 .5973$

\begin{abstract}
Hepatocellular carcinoma (HCC) is a predominantly male disease in which the androgen receptor (AR) serves an important pathogenic role in hepatocarcinogenesis. Fatty acid metabolism also contributes to hepatocarcinogenesis and is associated with the prognosis of cancer. The present study aimed to investigate the effects of the AR on fatty acid metabolism-associated gene expression in human hepatoma cell lines. AR-expression plasmids or control plasmids were transiently transfected into the human HCC cell lines Huh7 and HepG2. After $48 \mathrm{~h}$, cellular protein and RNA were extracted and the expression of AR was confirmed by western blotting. Complementary DNA was synthesized and subjected to a quantitative polymerase chain reaction-based array to examine the expression of 84 fatty acid metabolism-associated genes. Overexpression of AR significantly downregulated the expression of 11 fatty acid metabolism-associated genes in Huh7 cells and 35 in HepG 2 cells. The overexpression of AR also resulted in the upregulation of 6 fatty acid metabolism genes in HepG2 cells; however, it had no effect in Huh7 cells. Acyl-coenzyme A (CoA) thioesterase 7 and acyl-CoA oxidase 3 were downregulated in both cell lines. In conclusion, upregulation of $\mathrm{AR}$ via overexpression led to the disturbance of fatty acid metabolism-associated gene expression in human HCC cells. Therefore, the AR may serve a role in hepatocarcinogenesis via the regulation of hepatocellular fatty acid metabolism.
\end{abstract}

\section{Introduction}

Steroid sex hormones serve major roles as tumor promoters by promoting cell cycle progression in several types of cancer, including hepatocellular carcinoma (HCC) (1-3). The sex

Correspondence to: Dr Tatsuo Kanda, Department of Gastroenterology and Nephrology, Graduate School of Medicine, Chiba University, 1-8-1 Inohana, Chuo-ku, Chiba 260-8670, Japan

E-mail: kandat-cib@umin.ac.jp

Key words: androgen receptor, fatty acid, liver cancer hormone androgen and its associated androgen receptor (AR) are involved in the development of the lipogenic phenotype of cancer cells via the induction of proteins and enzymes, including fatty acid synthase (FASN), required for their autonomous nutrition (4).

A number of studies have implicated AR, a member of the nuclear steroid receptor superfamily, in hepatocarcinogenesis $(1,3,5-7)$. AR is a transcription factor activated via ligand-dependent and ligand-independent mechanisms (8). Cytochrome $\mathrm{P} 450$, transforming growth factor $\beta 1$, vascular endothelial growth factor A and heat shock protein family A member 5 are target genes of ARs in the liver $(3,7,9,10)$. Crosstalk between ARs and liver X receptor (nuclear receptor subfamily 1 group $\mathrm{H}$ member 2) has also been implicated in maintaining cholesterol homeostasis (11). It has also been demonstrated that the activity of ARs is coupled with the upregulation of FASN (4).

Abnormalities in cellular metabolism are closely associated with HCC occurrence and development $(4,12)$ and it has been demonstrated that FASN is highly expressed in a number of cancer types, including HCC (12). In HCC patients, abnormalities in plasma phospholipid fatty acid profiles are occasionally observed, which may be attributed to the alteration of intrinsic fatty acid metabolism caused by the cancer itself (13). Dietary fatty acids may be implicated in the risk of developing non-viral hepatitis-associated HCC (14); however, there is data to refute this hypothesis (13).

Fatty acid metabolism also serves a role in human carcinogenesis and is associated with a poor prognosis in HCC patients. The present study aimed to investigate the effects of AR expression on fatty acid metabolism-associated gene regulation in human HCC cell lines.

\section{Materials and methods}

Cell culture. The human HCC cell lines HepG2 and Huh7, as described previously (3), were purchased from the Japanese Collection of Research Bioresources Cell Bank (Ibaraki, Japan). The cells were maintained in Dulbecco's modified Eagle's medium supplemented with $10 \%$ fetal calf serum (Sigma-Aldrich; Merck KGaA, Darmstadt, Germany) and grown at $37^{\circ} \mathrm{C}$ with $5 \% \mathrm{CO}_{2}$. 
Transfection and RNA extraction. A total of $4 \times 10^{5}$ cells per well were placed in 6-well plates $24 \mathrm{~h}$ prior to transfection. The cells were transfected with $0.4 \mu \mathrm{g}$ human AR protein-expressing plasmid (pSG5-AR) or $0.4 \mu \mathrm{g}$ control vectors, which were kindly provided by Professor J.T. Isaacs of The Johns Hopkins University School of Medicine (Baltimore, MD, USA) (15), using Effectene Transfection Reagent (Qiagen GmbH, Hilden, Germany) according to the manufacturer's protocol. At $48 \mathrm{~h}$ post-transfection, cellular RNA was collected using RNeasy (Qiagen $\mathrm{GmbH}$ ), and RNA was stored at $-80^{\circ} \mathrm{C}$ until use. RNA quality was measured by a NanoDrop spectrophotometer (Thermo Fisher Scientific, Inc., Waltham, MA, USA).

Complementary DNA (cDNA) synthesis and quantiative polymerase chain reaction ( $q P C R$ ) arrays. cDNA synthesis was performed by an $\mathrm{RT}^{2}$ First Strand kit (Qiagen $\mathrm{GmbH}$ ) using $1 \mu \mathrm{g}$ of RNA per reaction. The cDNA synthesis reaction was incubated at $42^{\circ} \mathrm{C}$ for $15 \mathrm{~min}$ and then halted by heating at $95^{\circ} \mathrm{C}$ for $5 \mathrm{~min}$. A human fatty acid metabolism PCR array (no. PAHS-007Z; Qiagen GmbH) was carried out according to the manufacturer's protocol. Briefly, amplification of cDNA was monitored with SYBR-Green by quantitative PCR analysis. PCR was performed in $25 \mu 1$ of ROX PCR Master mix (Qiagen $\mathrm{GmbH}$ ) containing primers and $1 \mu \mathrm{l}$ of the reverse-transcription reaction mixture, using a 7300 Real-Time PCR system (Applied Biosystems; Thermo Fisher Scientific, Inc.) according to the manufacturer's protocol. The PCR reaction was performed as follows: $95^{\circ} \mathrm{C}$ for $1 \mathrm{~min}$, followed by 40 cycles of $95^{\circ} \mathrm{C}$ for $5 \mathrm{sec}$ and $60^{\circ} \mathrm{C}$ for $30 \mathrm{sec}$. The data analysis was performed using the comparative threshold cycle method (16) and analyzed using PCR Array Data Analysis Software v2.1 (http://www.sabiosciences.com/ pcrarraydataanalysis.php).

Western blot analysis. Cell lysates were prepared $48 \mathrm{~h}$ post transfection in 1X SDS sample buffer (Wako Pure Chemical Industries, Ltd., Osaka, Japan). Protein concentration was determined using the Bradford method. Cell lysate proteins ( $5 \mu \mathrm{g} / \mathrm{well}$ ) were separated by 5-20\% SDS-PAGE and transferred onto a nitrocellulose membrane (ATTO Corp., Tokyo, Japan). AR expression was analyzed by western blotting by incubating membranes with rabbit polyclonal antibodies against AR (no. sc-816; dilution, 1:1,000) or GAPDH (no. sc-25778; dilution, 1:2,000) (both from Santa Cruz Biotechnology Inc., Dallas, TX, USA) at $4^{\circ} \mathrm{C}$ for $16 \mathrm{~h}$. Membranes were then incubated with anti-rabbit IgG HRP-linked secondary antibody (no. 7074; dilution, 1:3,500; Cell Signaling Technology, Inc., Danvers, MA, USA) at room temperature for $1 \mathrm{~h}$. Proteins were visualized using an enhanced chemiluminescence western blotting substrate (GE Healthcare Life Sciences, Chalfont, UK) and scanned with an image analyzer (LAS-4000) and Image Gauge software (version 3.1) (both from Fujifilm, Tokyo, Japan). The relative quantity of protein was determined by normalizing to GAPDH.

Statistical analysis. For statistical analysis, two-tailed Student's $t$-tests were performed with DA Stats software version PAF01644 (NIFTY Corp.; Fujitsu). P $<0.05$ was considered to indicate a statistically significant difference.

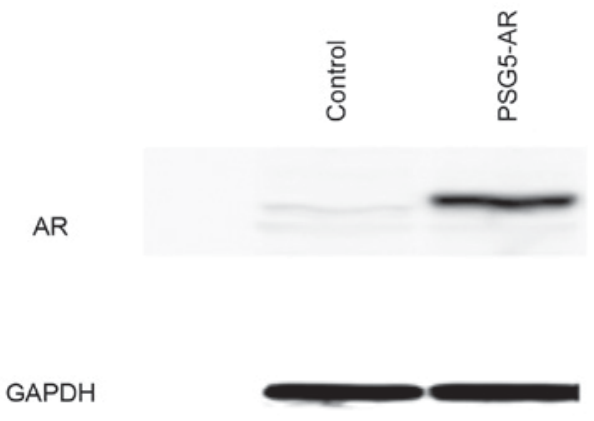

Figure 1. Overexpression of AR in HepG2 cells. Western blot analysis of AR and GAPDH expression level in HepG2 cells transiently transfected with the AR-expressing plasmid, pSG5-AR, or a control vector. AR, androgen receptor.

\section{Results}

Overexpression of AR in human HCC cells. Overexpression of AR was induced in HepG2 and Huh7 cells to validate hypotheses of the effect of AR on the regulation of fatty acid metabolism-associated gene expression. In HepG2 cells transiently transfected with pSG5-AR, overexpression of AR was confirmed by western blot analysis (Fig. 1), and AR overexpression in Huh7 cells transiently transfected with pSG5-AR was demonstrated by western blotting in a previous study (3).

Downregulated fatty acid metabolism-associated genes in AR-overexpressing HepG2 cells. Fatty acid metabolism-associated gene expression profiles were examined using a qPCR-based array. Expression levels between HepG2 cells transiently overexpressing AR and control vector-transfected HepG2 cells were compared. In HepG2 cells, AR overexpression significantly downregulated the expression of 35 fatty acid metabolism-associated genes, including 6 acyl-coenzyme A (CoA) dehydrogenases [acyl-CoA dehydrogenase family member (ACAD)10, acyl-CoA dehydrogenase, very long chain, acyl-CoA dehydrogenase, C-2 to C-3 short chain (ACADS), ACAD9, ACADS branched (B) and enoyl-CoA hydratase and 3-hydroxyacyl CoA dehydrogenase (EHHADH)], 2 acyl-CoA oxidases [acyl-CoA oxidase (ACOX) 1 and ACOX3], 4 acyl-CoA synthetases [acyl-CoA synthetase long-chain family member (ACSL)1, acyl-CoA synthetase bubblegum family member (ACSBG)2, ACSBG1 and ACSL4], 4 acyl-CoA thioesterases [acyl-CoA thioesterase (ACOT)8, ACOT1, ACOT2 and ACOT7], 4 carnitine transferases [carnitine palmitoyltransferase (CPT)2, carnitine $\mathrm{O}$-octanoyltranserase, carnitine $\mathrm{O}$-acetyltransferase, CPT1B], 5 other fatty acid metabolism-associated genes [aldehyde dehydrogenase 2 family (mitochondrial) (ALDH2), 2,4-dienoyl CoA reductase 2, peroxisomal, enoyl CoA hydratase, short chain, 1 , mitochondrial, enoyl-CoA $\delta$ isomerase 2 and methylmalonyl CoA mutase], 2 genes of fatty acid transport [CPT1A and solute carrier family 27 (fatty acid transporter), member 4], 4 genes of fatty acid biosynthesis regulation [protein kinase, cAMP-dependent, catalytic, $\alpha$ (PRKACA), protein kinase, AMP-activated, $\alpha 2$ catalytic subunit, protein kinase, AMP-activated, $\gamma 1$ non-catalytic subunit and protein kinase, AMP-activated, $\alpha 1$ catalytic 
Table I. Fatty acid metabolism-associated genes are significantly downregulated in androgen receptor-overexpressing HepG2 cells examined by polymerase chain reaction arrays.

\begin{tabular}{|c|c|c|c|}
\hline Symbol & Description & Fold change (vs. control) & P-value \\
\hline GPD1 & Glycerol-3-phosphatase dehydrogenase 1 (soluble) & 0.40 & 0.0259 \\
\hline ACAD 10 & Acyl-CoA dehydrogenase family, member 10 & 0.44 & 0.0135 \\
\hline LIPE & Lipase, hormone-sensitive & 0.50 & 0.0099 \\
\hline ACADVL & Acyl-CoA dehydrogenase, very long chain & 0.54 & 0.0018 \\
\hline CPT2 & Carnitine palmitoyltransferase 2 & 0.54 & 0.0181 \\
\hline CROT & Carnitine O-octanoyltranserase & 0.54 & 0.0069 \\
\hline CPT1A & Carnitine palmitoyltransferase 1A (liver) & 0.57 & 0.0039 \\
\hline ACSL1 & Acyl-CoA synthetase long-chain family member 1 & 0.57 & 0.0375 \\
\hline ACSBG2 & Acyl-CoA synthetase bubblegum family member 2 & 0.58 & 0.0096 \\
\hline ACADS & Acyl-CoA dehydrogenase, $\mathrm{C}-2$ to $\mathrm{C}-3$ short chain & 0.58 & 0.0448 \\
\hline ACAD9 & Acyl-CoA dehydrogenase family, member 9 & 0.59 & 0.0177 \\
\hline ACADSB & Acyl-CoA dehydrogenase, short/branched chain & 0.60 & 0.0475 \\
\hline ACOX1 & Acyl-CoA oxidase 1, palmitoyl & 0.60 & 0.0020 \\
\hline $\mathrm{ACOX} 3$ & Acyl-CoA oxidase 3 , pristanoyl & 0.60 & 0.0199 \\
\hline PRKACA & Protein kinase, cAMP-dependent, catalytic, $\alpha$ & 0.61 & 0.0012 \\
\hline PRKAA2 & Protein kinase, AMP-activated, $\alpha 2$ catalytic subunit & 0.61 & 0.0347 \\
\hline SLC27A4 & Solute carrier family 27 (fatty acid transporter), member 4 & 0.61 & 0.0428 \\
\hline BDH1 & 3-hydroxybutyrate dehydrogenase, type 1 & 0.61 & 0.0130 \\
\hline ACSBG1 & Acyl-CoA synthetase bubblegum family member 1 & 0.61 & 0.0233 \\
\hline EHHADH & Enoyl-CoA, hydratase/3-hydroxyacyl CoA dehydrogenase & 0.62 & 0.0212 \\
\hline ACSL4 & Acyl-CoA synthetase long-chain family member 4 & 0.63 & 0.0341 \\
\hline ACOT8 & Acyl-CoA thioesterase 8 & 0.63 & 0.0191 \\
\hline ACOT1 & Acyl-CoA thioesterase 1 & 0.64 & 0.0017 \\
\hline ALDH2 & Aldehyde dehydrogenase 2 family (mitochondrial) & 0.64 & 0.0088 \\
\hline PRKAG1 & Protein kinase, AMP-activated, $\gamma 1$ non-catalytic subunit & 0.64 & 0.0321 \\
\hline CRAT & Carnitine O-acetyltransferase & 0.66 & 0.0002 \\
\hline DECR2 & 2,4-dienoyl CoA reductase 2 , peroxisomal & 0.66 & 0.0412 \\
\hline ECHS1 & Enoyl CoA hydratase, short chain, 1, mitochondrial & 0.69 & 0.0368 \\
\hline $\mathrm{ECI} 2$ & Enoyl-CoA $\delta$ isomerase 2 & 0.70 & 0.0347 \\
\hline GK & Glycerol kinase & 0.72 & 0.0049 \\
\hline ACOT2 & Acyl-CoA thioesterase 2 & 0.73 & 0.0252 \\
\hline CPT1B & Carnitine palmitoyltransferase 1B (muscle) & 0.73 & 0.0038 \\
\hline PRKAA1 & Protein kinase, AMP-activated, $\alpha 1$ catalytic subunit & 0.76 & 0.0409 \\
\hline MUT & Methylmalonyl CoA mutase & 0.81 & 0.0453 \\
\hline ACOT7 & Acyl-CoA thioesterase 7 & 0.81 & 0.0098 \\
\hline
\end{tabular}

CoA, conenzyme A.

subunit], 1 gene of ketogenesis and ketone body metabolism (3-hydroxybutyrate dehydrogenase, type 1) and 3 genes of triacylglycerol metabolism [glycerol-3-phosphatase dehydrogenase 1 (soluble), lipase, hormone-sensitive and glycerol kinase; Table I].

Upregulated fatty acid metabolism-associated genes in AR-overexpressing HepG2 cells. In HepG2 cells, AR significantly upregulated the expression of 6 fatty acid metabolism-associated genes (Table II), including 3 fatty acid transports [Solute carrier family (SLC) 27A5, SLC27A2 and fatty acid binding protein 4 , adipocyte], 1 fatty acid biosynthesis regulator [protein kinase, AMP-activated, $\gamma 2$ non-catalytic subunit (PRKAG2)], 1 acyl-CoA thioesterase (ACOT9) and 1 other fatty acid metabolism-associated gene [pyrophosphatase (inorganic) 1 (PPA1)].

Upregulated and downregulated fatty acid metabolism-associated genes in AR-overexpressing Huh7 cells. In Huh7 cells, AR overexpression significantly downregulated the expression of the following 11 fatty acid metabolism-associated genes: 4 acyl-CoA synthetases [acyl-CoA synthetase medium-chain family member (ACSM) 2A, ACSL6, ACSL3 and ACSM3], 3 acyl-CoA thioesterases (ACOT7, ACOT9 and ACOT6), 1 acyl-CoA oxidase (ACOX3), 1 fatty acid transporter (SLC27A2), 1 fatty acid biosynthesis regulator (PRKAG2) 
Table II. Fatty acid metabolism-associated genes are significantly upregulated in androgen receptor-overexpressing HepG2 cells examined by polymerase chain reaction arrays.

\begin{tabular}{llcr}
\hline Symbol & \multicolumn{1}{c}{ Description } & Fold change (vs. control) & P-value \\
\hline FABP4 & Fatty acid binding protein 4, adipocyte & 2.25 & 0.0015 \\
SLC27A2 & Solute carrier family 27 (fatty acid transporter), member 2 & 1.51 & 0.0167 \\
SLC27A5 & Solute carrier family 27 (fatty acid transporter), member 5 & 1.40 & 0.0156 \\
ACOT9 & Acyl-CoA thioesterase 9 & 1.25 & 0.0216 \\
PPA1 & Pyrophosphatase (inorganic) 1 & 1.23 & 0.0162 \\
PRKAG2 & Protein kinase, AMP-activated, $\gamma$ 2 non-catalytic subunit & 1.20 & 0.0331 \\
\hline
\end{tabular}

CoA, conenzyme A.

Table III. Fatty acid metabolism-associated genes are significantly downregulated in androgen receptor-overexpressing Huh7 cells.

\begin{tabular}{llcr}
\hline Symbol & \multicolumn{1}{c}{ Description } & Fold change (vs. control) & P-value \\
\hline ACOX3 & Acyl-CoA oxidase 3, pristanoyl & 0.62 & 0.0180 \\
PRKAG2 & Protein kinase, AMP-activated, $\gamma$ 2 non-catalytic subunit & 0.65 & 0.0402 \\
ACSM3 & Acyl-CoA synthetase medium-chain family member 3 & 0.66 & 0.0249 \\
SLC27A2 & Solute carrier family 27 (fatty acid transporter), member 2 & 0.69 & 0.0387 \\
BDH2 & 3-hydroxybutyrate dehydrogenase, type 2 & 0.69 & 0.0015 \\
ACSL3 & Acyl-CoA synthetase long-chain family member 3 & 0.87 & 0.0127 \\
ACOT6 & Acyl-CoA thioesterase 6 & 0.88 & 0.0020 \\
ACSL6 & Acyl-CoA synthetase long-chain family member 6 & 0.89 & 0.0052 \\
ACOT9 & Acyl-CoA thioesterase 9 & 0.91 & 0.0434 \\
ACOT7 & Acyl-CoA thioesterase 7 & 0.92 & 0.0480 \\
ACSM2A & Acyl-CoA synthetase medium-chain family member 2A & 0.93 & 0.0018
\end{tabular}

CoA, conenzyme A.

and 1 regulator of ketogenesis and ketone body metabolism (3-hydroxybutyrate dehydrogenase, type 2; Table III). However, AR overexpression did not significantly upregulate the expression of any fatty acid metabolism-associated genes in Huh7 cells.

\section{Discussion}

The present study demonstrated that overexpression of AR alters fatty acid metabolism-associated gene expression in human HCC cell lines. Previous studies $(17,18)$ demonstrated that in patients with HCC, ACADSB and EHHADH expression are downregulated in tissue isolated from cirrhotic livers compared with control tissues from healthy patients. Acyl-CoA oxidase deficient-mice spontaneously develop HCC (19). Li et al (20) reported that cluster of differentiation (CD) 147 expression significantly contributed to the reprogramming of fatty acid metabolism in HCC cells, and that CD147 downregulated CPT1A and ACOX1 by activating the p38 mitogen-activated protein kinase signaling pathway to inhibit fatty acid $\beta$-oxidation. A previous study in phosphatase and tensin homolog null nonalcoholic steatohepatitis liver tissue isolated from mice revealed that ACSL1 expression is decreased, and is almost non-existent in tumors isolated from these livers (21). A recent study (22) demonstrated that the presence of a specific mutation in ALDH2 (E487 K) led to increased protein turnover and promoted murine hepatocarcinogenesis. The presence of the DnaJ heat shock protein family (Hsp40) member B1-PRKACA chimeric transcript in fibrolamellar HCC suggests that this genetic alteration contributes to tumor pathogenesis (23). Therefore, the present study supports previous reports (17-23) (Table I).

Sakabe et al (24) reported that PRKAG2 enhances the effect of interferon (IFN)- $\alpha$ /fluorouracil (5-FU) and serves as a prognostic marker for IFN- $\alpha / 5$-FU therapy in HCC. The present study demonstrated that AR significantly upregulated the expression of PRKAG2 (Table II). ACOT7 has been implicated in the regulation of neuronal fatty acid metabolism to prevent neurotoxicity (25), and ACOX3 is expressed at particularly low levels in the liver (26). Notably, ACOT7 and ACOX3 were downregulated by the overexpression of AR in HepG2 and Huh7 cells (Tables I and III). These results support the results of a previous study by Bolton et al (27) that implicated AR in the regulation of cellular lipid metabolism.

In general, cancer cells synthesize a high amount of fatty acids via increased expression, and subsequent activity, of 
lipogenic enzymes to fulfill their high energy requirements and to maintain cell membranes for growth $(4,28,29)$. In patients with HCC, the alterations in lipid and lipoprotein metabolism can be clearly observed (30).

AR has been demonstrated to be associated with hepatocarcinogenesis and HCC development $(1,3,5-7,9,10)$. The present study revealed that AR could serve a role in hepatocarcinogenesis via the regulation of hepatocellular fatty acid metabolism. Hepatitis $\mathrm{C}$ virus (HCV) augments AR-mediated signaling (7) and replicates in hepatocytes using lipid droplets, which are used for the storage of neutral lipids (31). In a previous study, eradication of $\mathrm{HCV}$ using an interferon-free antiviral regimen resulted in rapid changes in the metabolic signaling pathway in the liver, which suggested that there was a direct effect of HCV replication on lipid homeostasis (32); however, further studies are required to determine potential indirect effects of $\mathrm{HCV}$ on lipid homeostasis. Hepatitis B virus (HBV) has also been revealed to augment AR-mediated signaling $(5,6)$ and to induce hepatic steatosis by influencing fatty acid metabolism (33). HBV serves a more minor role in hepatic steatosis compared with HCV (34).

In conclusion, improving the understanding of the association between AR and fatty acid metabolism in hepatocarcinogenesis and the development of HCC may aid in the creation of novel therapeutic and prevention strategies.

\section{Acknowledgements}

The authors would like to thank Professor J.T. Isaacs (The Johns Hopkins University School of Medicine, Baltimore, MD, USA) for providing the plasmid pSG5-AR. The present work was supported by grants from the Ministry of Health, Labour and Welfare of Japan. This study was partially supported by the Research Program on Hepatitis from the Japan Agency for Medical Research and Development (AMED).

\section{References}

1. Nagasue N, Chang YC, Hayashi T, Galizia G, Kohno H, Nakamura T and Yukaya H: Androgen receptor in hepatocellular carcinoma as a prognostic factor after hepatic resection. Ann Surg 209: 424-427, 1989.

2. Okitsu K, Kanda T, Imazeki F, Yonemitsu Y, Ray RB, Chang C and Yokosuka O: Involvement of interleukin- 6 and androgen receptor signaling in pancreatic cancer. Genes Cancer 1: 859-867, 2010.

3. Jiang $\mathrm{X}$, Kanda T, Nakamoto S, Miyamura T, Wu S and Yokosuka O: Involvement of androgen receptor and glucose-regulated protein $78 \mathrm{kDa}$ in human hepatocarcinogenesis. Exp Cell Res 323: 326-336, 2014

4. Rochefort $\mathrm{H}$ and Chalbos D: The role of sex steroid receptors on lipogenesis in breast and prostate carcinogenesis: A viewpoint. Horm Cancer 1: 63-70, 2010.

5. Chiu CM, Yeh SH, Chen PJ, Kuo TJ, Chang CJ, Chen PJ, Yang WJ and Chen DS: Hepatitis B virus X protein enhances androgen receptor-responsive gene expression depending on androgen level. Proc Natl Acad Sci USA 104: 2571-2578, 2007.

6. Zheng Y, Chen WL, Ma WL, Chang C and Ou JH: Enhancement of gene transactivation activity of androgen receptor by hepatitis B virus X protein. Virology 363: 454-461, 2007.

7. Kanda T, Steele R, Ray R and Ray RB: Hepatitis C virus core protein augments androgen receptor-mediated signaling. J Virol 82: 11066-11072, 2008.

8. Ueda T, Bruchovsky $\mathrm{N}$ and Sadar MD: Activation of the androgen receptor $\mathrm{N}$-terminal domain by interleukin- 6 via MAPK and STAT3 signal transduction pathways. J Biol Chem 277: 7076-7085, 2002.
9. Gehlhaus M, Schmitt N, Volk B and Meyer RP: Antiepileptic drugs affect neuronal androgen signaling via a cytochrome P450-dependent pathway. J Pharmacol Exp Ther 322: 550-559, 2007.

10. Yoon G, Kim JY, Choi YK, Won YS and Lim IK: Direct activation of TGF-beta1 transcription by androgen and androgen receptor complex in Huh7 human hepatoma cells and its tumor in nude mice. J Cell Biochem 97: 393-411, 2006.

11. Krycer JR and Brown AJ: Cross-talk between the androgen receptor and the liver $\mathrm{X}$ receptor: Implications for cholesterol homeostasis. J Biol Chem 286: 20637-20647, 2011.

12. Hao Q, Li T, Zhang X, Gao P, Qiao P, Li S and Geng Z: Expression and roles of fatty acid synthase in hepatocellular carcinoma. Oncol Rep 32: 2471-2476, 2014.

13. Qiu JF, Zhang KL, Zhang XJ, Hu YJ, Li P, Shang CZ and Wan JB: Abnormalities in plasma phospholipid fatty acid profiles of patients with hepatocellular carcinoma. Lipids 50: 977-985, 2015.

14. Koh WP, Dan YY, Goh GB, Jin A, Wang R and Yuan JM: Dietary fatty acids and risk of hepatocellular carcinoma in the Singapore Chinese health study. Liver Int 36: 893-901, 2016.

15. Litvinov IV, Chang C and Isaacs JT: Molecular characterization of the commonly used human androgen receptor expression vector, pSG5-AR. Prostate 58: 319-324, 2004.

16. Livak KJ and Schmittgen TD: Analysis of relative gene expression data using real-time quantitative PCR and the 2(-Delta Delta C(T)) method. Methods 25: 402-408, 2001.

17. Schröder PC, Segura V, Riezu JI, Sangro B, Mato JM, Prieto J, Santamaría E and Corrales FJ: A signature of six genes highlights defects on cell growth and specific metabolic pathways in murine and human hepatocellular carcinoma. Funct Integr Genomics 11: 419-429, 2011.

18. Suto K, Kajihara-Kano H, Yokoyama Y, Hayakari M, Kimura J, Kumano T, Takahata T, Kudo H and Tsuchida S: Decreased expression of the peroxisomal bifunctional enzyme and carbonyl reductase in human hepatocellular carcinomas. J Cancer Res Clin Oncol 125: 83-88, 1999.

19. Meyer K, Lee JS, Dyck PA, Cao WQ, Rao MS, Thorgeirsson SS and Reddy JK: Molecular profiling of hepatocellular carcinomas developing spontaneously in acyl-CoA oxidase deficient mice: Comparison with liver tumors induced in wild-type mice by a peroxisome proliferator and a genotoxic carcinogen. Carcinogenesis 24: 975-984, 2003.

20. Li J, Huang Q, Long X, Zhang J, Huang X, Aa J, Yang H, Chen Z and Xing J: CD147 reprograms fatty acid metabolism in hepatocellular carcinoma cells through Akt/mTOR/SREBP1c and P38/PPAR $\alpha$ pathways. J Hepatol 63: 1378-1389, 2015.

21. Muir K, Hazim A, He Y, Peyressatre M, Kim DY, Song X and Beretta L: Proteomic and lipidomic signatures of lipid metabolism in NASH-associated hepatocellular carcinoma. Cancer Res 73: 4722-4731, 2013.

22. Jin S, Chen J, Chen L, Histen G, Lin Z, Gross S, Hixon J, Chen Y, Kung C, Chen Y, et al: ALDH2(E487K) mutation increases protein turnover and promotes murine hepatocarcinogenesis. Proc Natl Acad Sci USA 112: 9088-9093, 2015.

23. Honeyman JN, Simon EP, Robine N, Chiaroni-Clarke R, Darcy DG, Lim II, Gleason CE Murphy JM, Rosenberg BR Teegan L, et al: Detection of a recurrent DNAJB1-PRKACA chimeric transcript in fibrolamellar hepatocellular carcinoma. Science 343: 1010-1014, 2014.

24. Sakabe T, Tsuchiya H, Kanki K, Azumi J, Gonda K, Mizuta Y, Yamada D, Wada H, Shomori K, Nagano H and Shiota G: Identification of the genes chemosensitizing hepatocellular carcinoma cells to interferon- $\alpha / 5$-fluorouracil and their clinical significance. PLoS One 8: e56197, 2013.

25. Ellis JM, Wong GW and Wolfgang MJ: Acyl coenzyme A thioesterase 7 regulates neuronal fatty acid metabolism to prevent neurotoxicity. Mol Cell Biol 33: 1869-1882, 2013.

26. Zha S, Ferdinandusse S, Hicks JL, Denis S, Dunn TA, Wanders RJ, Luo J, De Marzo AM and Isaacs WB: Peroxisomal branched chain fatty acid beta-oxidation pathway is upregulated in prostate cancer. Prostate 63: 316-323, 2005.

27. Bolton EC, So AY, Chaivorapol C, Haqq CM, Li H and Yamamoto KR: Cell- and gene-specific regulation of primary target genes by the androgen receptor. Genes Dev 21: 2005-2017, 2007.

28. Baron A, Migita T, Tang D and Loda M: Fatty acid synthase: A metabolic oncogene in prostate cancer? J Cell Biochem 91: 47-53, 2004. 
29. Mounier C, Bouraoui L and Rassart E: Lipogenesis in cancer progression (review). Int J Oncol 45: 485-492, 2014.

30. Jiang J, Nilsson-Ehle P and Xu N: Influence of liver cancer on lipid and lipoprotein metabolism. Lipids Health Dis 5: 4, 2006.

31. Miyanari Y, Atsuzawa K, Usuda N, Watashi K, Hishiki T, Zayas M, Bartenschlager R, Wakita T, Hijikata $M$ and Shimotohno K: The lipid droplet is an important organelle for hepatitis C virus production. Nat Cell Biol 9: 1089-1097, 2007.

32. Meissner EG, Lee YJ, Osinusi A, Sims Z, Qin J, Sturdevant D, McHutchison J, Subramanian M, Sampson M, Naggie S, et al: Effect of sofosbuvir and ribavirin treatment on peripheral and hepatic lipid metabolism in chronic hepatitis $\mathrm{C}$ virus, genotype 1-infected patients. Hepatology 61: 790-801, 2015.
33. Wu YL, Peng XE, Zhu YB, Yan XL, Chen WN and Lin X: Hepatitis B virus $X$ protein induces hepatic steatosis by enhancing the expression of liver fatty acid binding protein. J Virol 90: 1729-1740, 2015.

34. Haga Y, Kanda T, Sasaki R, Nakamura M, Nakamoto S and Yokosuka O: Nonalcoholic fatty liver disease and hepatic cirrhosis: Comparison with viral hepatitis-associated steatosis. World J Gastroenterol 21: 12989-12995, 2015. 